Check for updates

Cite this: RSC Adv., 2017, 7, 26697

\title{
Computational investigation on the large energy gap between the triplet excited-states in acenes
}

\author{
Y. Y. Pan, (D) *ab J. Huang, ${ }^{a}$ Z. M. Wang, ${ }^{a}$ D. W. Yu, ${ }^{a}$ B. Yang ${ }^{\star b}$ and Y. G. Ma (D) ${ }^{c}$
}

The large energy gap between the two triplet excited-states in acenes has a huge impact on their optical and electronic properties. Accurate calculation and full use of this gap have always been a major challenge in the field of organic semiconductor materials. In the present study, we focus on the precise description of the large gap between the $T_{1}$ and $T_{2}$ states, and taking a series of acenes (benzene, naphthalene, anthracene, tetracene, and pentacene) as examples, investigate their excited state behavior to verify the energy gap structure. The results show that the symmetry of the transition molecular orbital and the excited state properties have a great influence on the transition energy, and may be the main cause of the large energy gap.

Received 2nd March 2017 Accepted 4th May 2017

DOI: $10.1039 / c 7 r a 02559 a$

rsc.li/rsc-advances mechanism, the energy of the $\mathrm{T}_{1}$ state is greater than the $\mathrm{S}_{1}$ but less than $\mathrm{T}_{2}\left(E_{\mathrm{S} 1}<2 E_{\mathrm{T} 1}<E_{\mathrm{T} 2}\right)$, that is, there are energy gaps between the $S_{1}, T_{1}$ and $T_{2}$, as shown in Fig. $1(c) .{ }^{11}$ The third example is singlet fission (SF) in which two triplet excitations are produced from an excited singlet. Suitable candidates for SF should have $S_{1}$ state energy more than twice the $T_{1}$ energy and for the efficient formation of triplets by singlet fission, it is important to ensure that neither $2 E_{\mathrm{T} 1}-E_{\mathrm{S} 1}$ nor $2 E_{\mathrm{T} 1}-E_{\mathrm{T} 2}$ are distinctly positive, as shown in Fig. 1 (d). ${ }^{12-14}$ The above three examples show that the energy gap between the triplet states can slow down the IC rate which is comparable with the ISC rate. This will give rise to a change in the resultant ratio of excitons generated between the singlet and triplet state in the EL device, which further causes a change in fluorescent efficiency.

Among the many electroluminescent materials, due to their simple structure, easy synthesis and high luminous efficiency, acenes have received extensive attention as potential candidate materials for TTA, hot exciton and singlet fission; thus the study of acenes triplet excited-state has also gradually increased. ${ }^{15-21}$ For instance, Lewis and Kasha, ${ }^{22}$ McClure, ${ }^{23}$ Hunziker, ${ }^{24,25}$ and Meyer et $a l^{26}$ have probed experimentally the triplet states of naphthalene and the lowest triplet state in longer stable oligomers has also been determined experimentally. ${ }^{27-31}$ However, the energy of the higher triplet states and the energy gap between them have not been given any attention both experimentally and theoretically.

In the present study, we first aim to ascertain the accuracy of the method applied to describe the energy level of the triplet state, and on this basis, we further focus on the large transition energy gap between the $T_{1}$ and $T_{2}$ state taking a series of acenes (benzene, naphthalene, anthracene, tetracene, and pentacene) (as shown in Scheme 1) as examples to investigate the transition properties of their triplet excited states to explore the huge

\footnotetext{
${ }^{a}$ School of Petrochemical Engineering, Shenyang University of Technology, 30 Guanghua Street, Liaoyang, 111003, P. R. China. E-mail: pyy39518768@163.com; Fax: +86-419-5319409; Tel: +86-419-5319409

${ }^{5}$ State Key Laboratory of Supramolecular Structure and Materials, Jilin University, Changchun, 130012, P. R. China. E-mail: yangbing@jlu.edu.cn; Fax: +86-43185168502; Tel: +86-431-85193421

'State Key Laboratory of Luminescent Materials and Devices, Institute of Polymer Optoelectronic Materials and Devices, South China University of Technology, Guangzhou, 510640, P. R. China
} 
(a)

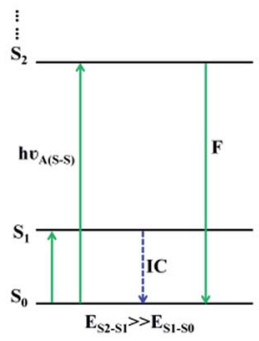

(c)

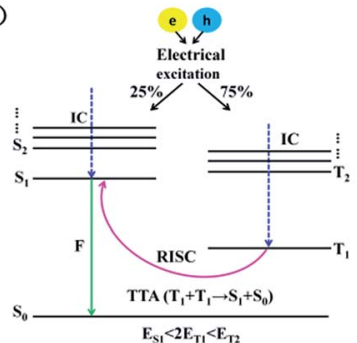

(b)

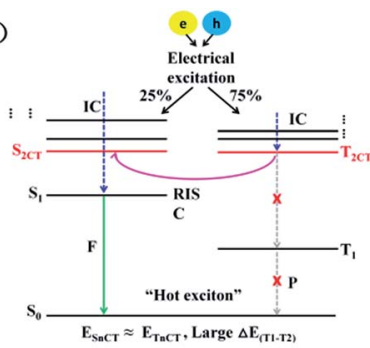

(d)

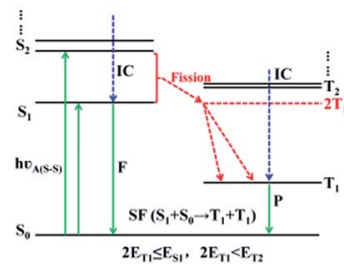

Fig. 1 Simple schematic of the exciton decay and the electroluminescence process of (a) azulene molecules; (b) hot exciton mechanism; (c) TTA mechanism; and (d) SF mechanism. Where, S: singlet state; $\mathrm{T}$ : triplet state; $F$ : fluorescence; $P$ : phosphorescence; $K_{\mathrm{IC}}$ : internal conversion rate; $K_{\mathrm{RISC}}$ : reverse intersystem crossing rate; $\mathrm{CT}$ : charge-transfer state; and $\Delta E_{\mathrm{ST}}$ : singlet-triplet energy splitting.
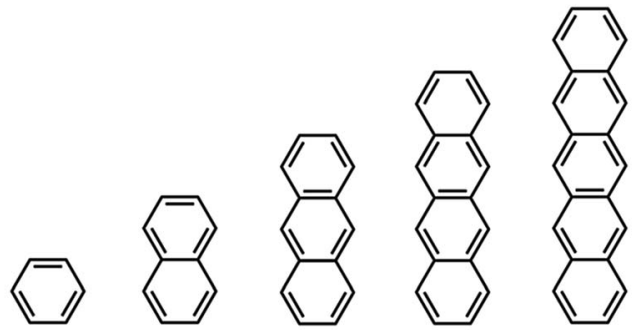

Benzene Naphthalene Anthracene Tetracene

Pentacene

Scheme 1 Representation of the acenes investigated herein.

resulting $\Delta E_{\mathrm{T} 1-\mathrm{T} 2}$. The study of the excited state behaviour of these molecules to verify their high level triplet excited state energy gap structure is very helpful for a rational molecular design to adjust and control the gap between the excited states with the aim of developing new ideas on the adjustable energy gap of high performance organic photoelectric functional materials.

\section{Methodology and computational details}

As is known, the ground-state electronic structures for most chromophores can be conveniently obtained using various quantum chemistry computational methods. However, it is difficult to calculate the electronic structures of the electronic excited state for molecular systems, especially the triplet state. Nowadays, the equation of motion coupled cluster with single and double excitation model ${ }^{32}$ (EOM-CCSD) methods and time-dependent density functional theory ${ }^{33-38}$ (TD-DFT) are very popular computational methods to determine excitedstate electronic structures. TD-DFT is the most widely-used method to describe the excited-state properties of medium to large molecular systems. The EOM-CCSD method takes into account both single and double substitutions and can give relatively accurate results, but the calculation cost is very high; whereas TD-DFT is a more cost-effective method to calculate the excited-state. In the present study, in order to explore the energy gap between the triplet excited states of acenes, the excited state energy needs to be calculated accurately first. To assess the performances of the TD-DFT approaches, we tried to cover a broad and diverse selection of functionals. We use the typical acenes molecule anthracene, tetracene and pentacene (Scheme 1) as examples to investigate the most suitable functionals. The performance of 2 local functionals $\left(\mathrm{SVWN}^{39}\right.$ and $\left.\mathrm{PBE}^{40}\right), 7$ hybrid functionals (BLYP $(0 \% \mathrm{HF}){ }^{41}$ B3LYP $(20 \% \mathrm{HF}),{ }^{42}$ PBE0 $(25 \% \mathrm{HF}),{ }^{43} \mathrm{BMK}(42 \% \mathrm{HF}),{ }^{44} \mathrm{BH}$ and HLYP $(50 \% \mathrm{HF}),{ }^{45} \mathrm{M} 06-2 \mathrm{X}(56 \% \mathrm{HF}),{ }^{46}$ and M06HF $(100 \%$ $\mathrm{HF}),{ }^{47}$ ) and 2 long-range-corrected functionals CAM-B3LYP ${ }^{48}$ and $\omega \mathrm{B} 97 \mathrm{X}^{49}$ in combination with $6-31+\mathrm{G}(\mathrm{d}, \mathrm{p})$ basis sets ${ }^{50}$ is explored. The EOM-CCSD method is also included for comparison purposes.

In this part, we focus on describing the transition energy level of the triplet states $\left(T_{1}\right.$ and $\left.T_{2}\right)$ accurately. As shown in Fig. 2, we used different methods and functionals as abscissa, and the difference between the experimental value as the ordinate for mapping histograms.${ }^{51}$ On the whole, each functional of the DFT method shows a tendency to gradually underestimate the transition energy as the conjugate length increases. When the conjugate length is small (anthracene), the difference between the calculated values of $T_{1}$ and $T_{2}$ and the experimental values is not as large as the whole, where the maximum value is $0.83 \mathrm{eV}$ and the minimum value is only $0.01 \mathrm{eV}$, among which the BLYP, B3LYP and $\omega$ B97X functionals are controlled within the range of $0.1 \mathrm{eV}$. When the conjugate length increases to tetracene, the difference increases and the maximum value reaches $-1.96 \mathrm{eV}$, where the BLYP and $\omega$ B97X functionals remain within the range of 0.1 . When the conjugate length continues to increase to pentacene, the difference is further increased, where the maximum value is $-2.08 \mathrm{eV}$, as calculated by the BH and HLYP functional. As can be seen in Fig. 2(e) and (f), the difference values are systematically negative, which indicates that the TD-DFT functional tends to underestimate the transition energies in the relatively long conjugate systems. Only the $\omega \mathrm{B} 97 \mathrm{X}$ functional continues to maintain a difference of less than 0.1. This situation may be due to the conjugate length growth, where the distance of the electron transition increases, and similar to the charge transfer process, the traditional local functionals and most hybrid functionals underestimate the transition energy with an increase in conjugate length. However, the $\omega$ B97X functional optimizes the range separation parameter $\omega$ through the self-consistent restriction system, which aims at physically motivated correction for the long-range Coulomb interaction. This functional can be established to enable the accurate and quantitative description of the intramolecular charge-transfer 
(a)

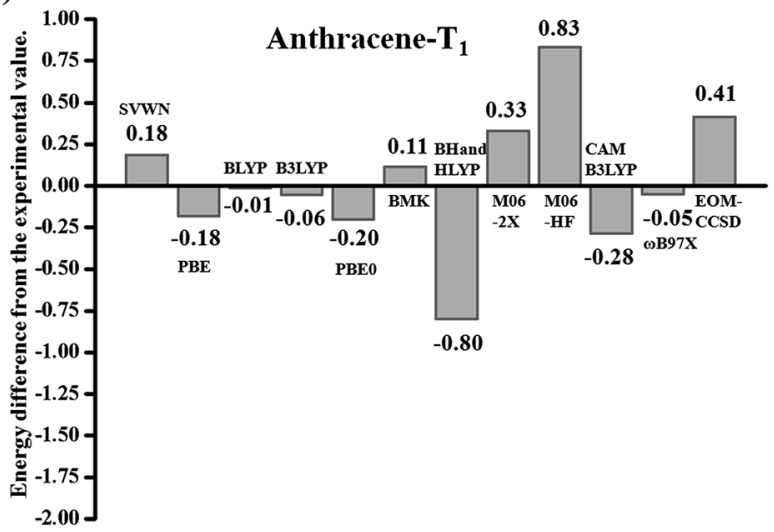

(c)

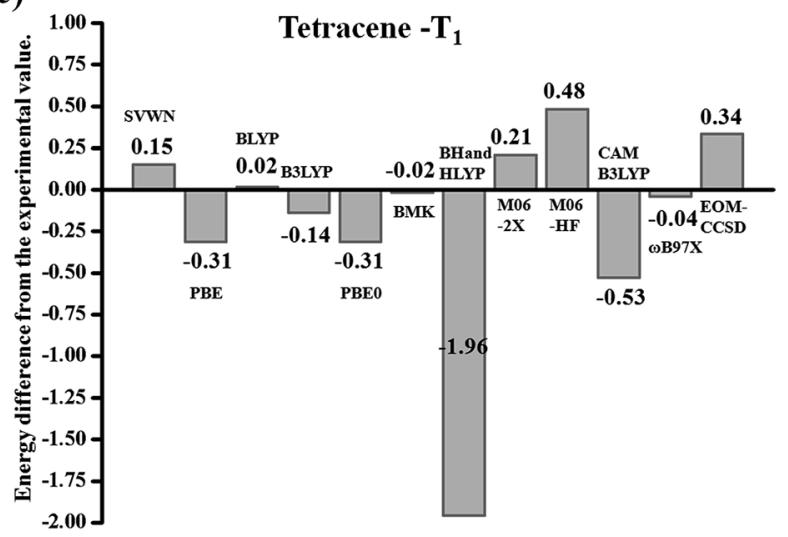

(e)

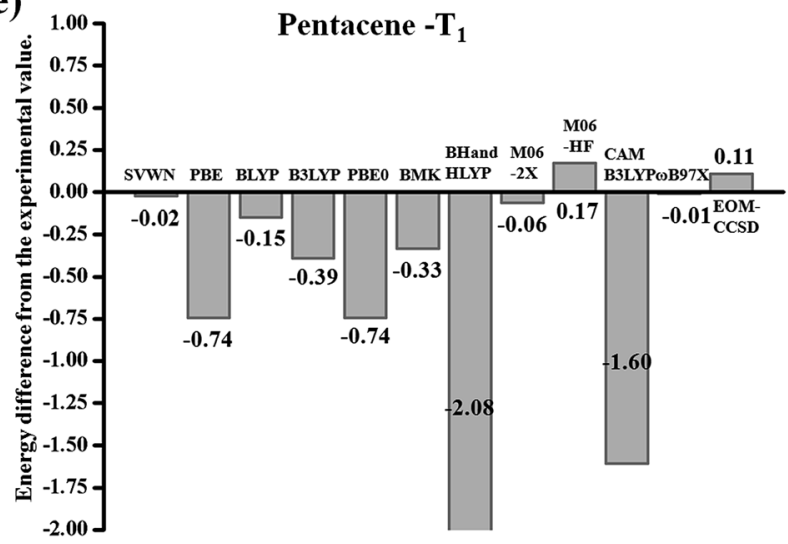

(b)

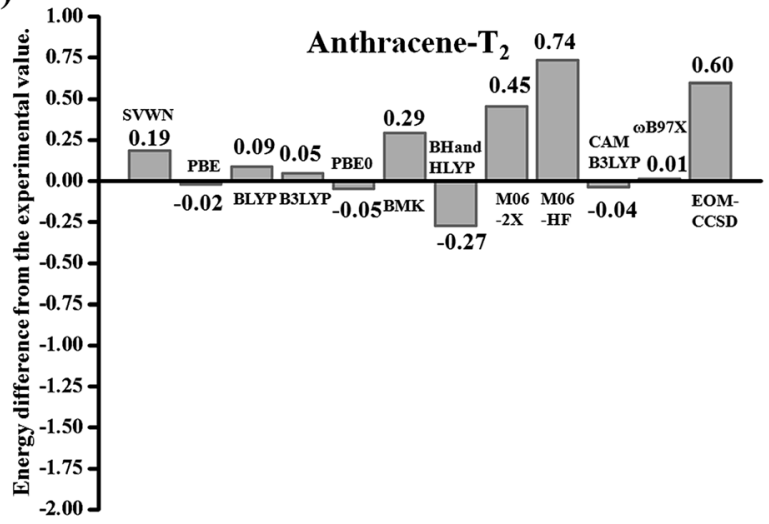

(d)

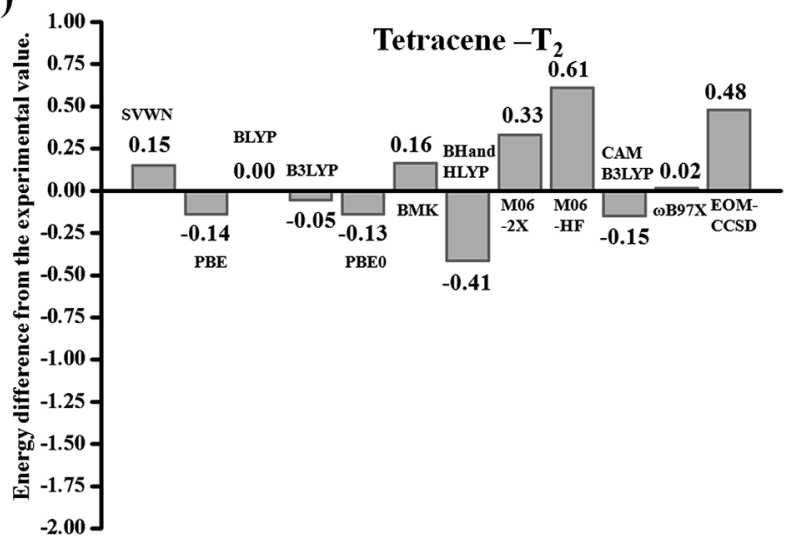

(f)

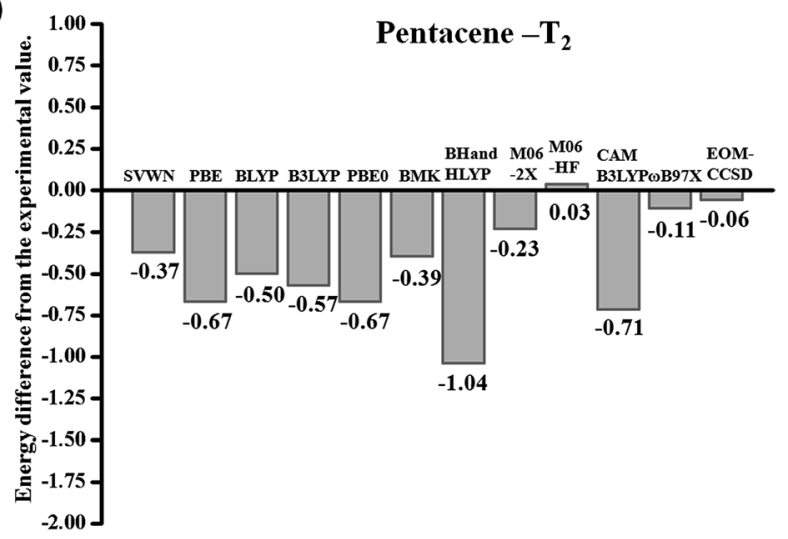

Fig. 2 Histograms of the difference from the experimental value for the triplet vertical excitation energies (eV) calculated using TD-DFT and the EOM-CCSD method with respect to the acene: (a) $T_{1}$ for anthracene; (b) $T_{2}$ for anthracene; (c) $T_{1}$ for tetracene; (d) $T_{2}$ for tetracene; (e) $T_{1}$ for pentacene and (f) $T_{2}$ for pentacene.

(CT) state. At the same time, the EOM-CCSD method also exhibits the trend that with conjugate length growth the transition energy gradually goes from being overvalued to underestimated. Considering the computational accuracy and cost, the TD-DFT/ $\omega$ B 97X method was finally chosen to describe the excited-state properties of acenes. Earlier benchmarks have shown that different functionals have almost no effect on the geometries of the ground states and the minimum structures are similar in accuracy to the structures obtained from experiment. ${ }^{52}$ Herein, all ground state structures were optimized using the B3LYP functional combined with the $6-31+\mathrm{G}(\mathrm{d}, \mathrm{p})$ basis set. The optimized structures were confirmed to be local energy minima by calculating vibrational frequencies. All DFT/TD-DFT and EOMCCSD calculations were performed with Gaussian09.D.01 Revision. ${ }^{53}$ 


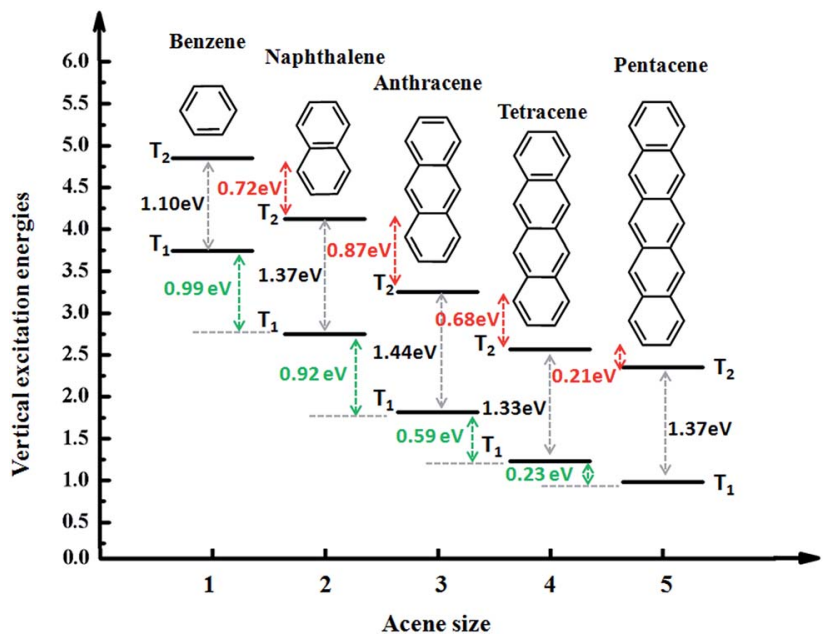

Fig. 3 Energy diagrams of the acenes (benzene, naphthalene, anthracene (An), tetracene, and pentacene).

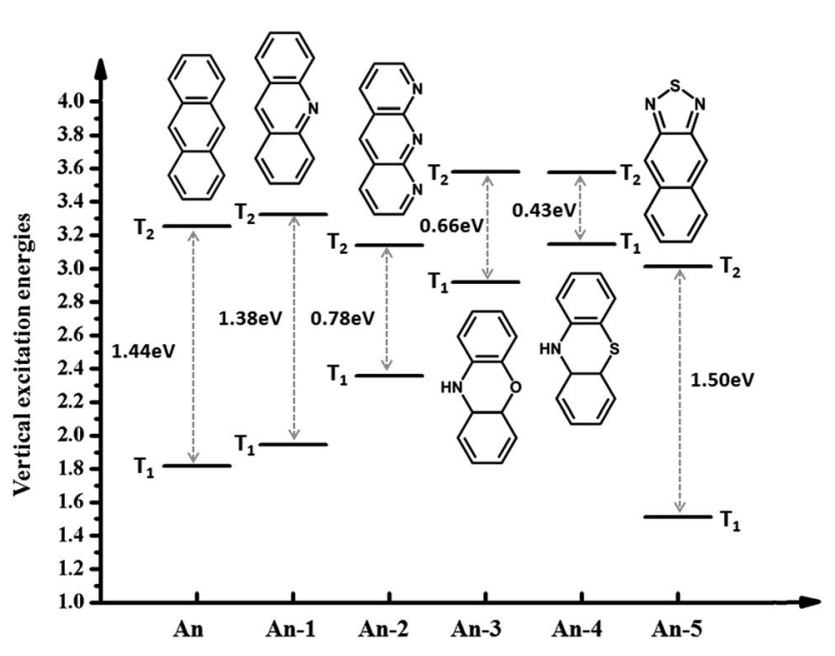

Fig. 4 Energy diagrams of anthracene derivatives of the first two triplet excited-states from calculation.

\section{Results and discussion}

3.1 Transition energy gap between $T_{1}$ and $T_{2}\left(\Delta E_{T 1-T 2}\right)$

We take the conjugate length (number of benzene ring) as the abscissa and triplet excitation energy as the ordinate in drawing presented in Fig. 3. Overall, with the growth of the conjugated length and the excitation energies of $\mathrm{T}_{1}$ and $\mathrm{T}_{2}$ show a downward trend, which can be explained by the fact that the excitation energy of the triplet states is associated with the conjugate length, and the longer the length the lower the transition energy. However, there is a special phenomenon that from benzene to anthracene $T_{1}$ decreases by about $1.0 \mathrm{eV}$ for each benzene added and it decreases by only about $0.2 \mathrm{eV}$ from tetracene to pentacene. At the same time, the excitation energy of $\mathrm{T}_{2}$ reduces by a similar value of about $0.8 \mathrm{eV}$ from benzene to anthracene, until from tetracene to pentacene the gap between $\mathrm{T}_{2}$ is reduced to $0.2 \mathrm{eV}$. Thus, the trend of the gap between $\mathrm{T}_{1}$ and $\mathrm{T}_{2}\left(\Delta E_{\mathrm{T} 1-\mathrm{T} 2}\right)$ first increases (from $1.10 \mathrm{eV}$ to $1.44 \mathrm{eV}$ ) then decreases (from $1.44 \mathrm{eV}$ to $1.33 \mathrm{eV}$ ) and finally stabilizes (from $1.33 \mathrm{eV}$ to $1.37 \mathrm{eV}$ ).

Anthracene has the largest $\Delta E_{\mathrm{T} 1-\mathrm{T} 2}$ and benzene has the smallest $\Delta E_{\mathrm{T} 1-\mathrm{T} 2}$. Is the large $\Delta E_{\mathrm{T} 1-\mathrm{T} 2}$ related to its structural characteristics? To facilitate a comparison, we chose a series of common anthracene derivatives, for which their triplet excitation energy was calculated using the TD-DFT/ $\omega$ B97X method, as presented in Fig. 4. The results show that due to the introduction of heteroatoms, the energy changes a lot, where $\Delta E_{\mathrm{T} 1-\mathrm{T} 2}$ both increases and decreases, which indicates that the cause of the large $\Delta E_{\mathrm{T} 1-\mathrm{T} 2}$ is the structure and other reasons. In the next section we will discuss the cause of the large more $\Delta E_{\mathrm{T} 1-\mathrm{T} 2}$ by analyzing the nature of the transitional or excited states.

\subsection{The molecular orbitals (MOs)}

We analyzed the transition configurations of the $T_{1}$ and $T_{2}$ excited-state of the acenes. As shown in Table 1 , the $\mathrm{T}_{1}$ transition is mainly contributed from the highest occupied molecular orbital (HOMO) to the lowest unoccupied molecular orbital

Table 1 MOs character and symmetry of the acenes (benzene, naphthalene, anthracene (An), tetracene, and pentacene)

\begin{tabular}{|c|c|c|c|c|}
\hline & MOs character and coefficient & Orbital symmetry & $\begin{array}{l}\text { Transition probability } \\
\text { (YES or NO) }\end{array}$ & $\Delta E_{\mathrm{T} 1-\mathrm{T} 2}(\mathrm{eV})$ \\
\hline \multirow[t]{2}{*}{ Benzene } & $\mathrm{T}_{1} \mathrm{HOMO} \rightarrow$ LUMO 0.48 & $\mathrm{e} 1 \mathrm{~g} \rightarrow \mathrm{e} 2 \mathrm{u}$ & YES & \multirow[t]{2}{*}{1.10} \\
\hline & $\mathrm{T}_{3} \mathrm{HOMO}-1 \rightarrow \mathrm{LUMO}+10.47$ & $\mathrm{e} 1 \mathrm{~g} \rightarrow \mathrm{e} 2 \mathrm{u}$ & YES & \\
\hline \multirow[t]{2}{*}{ Naphthalene } & $\mathrm{T}_{1} \mathrm{HOMO} \rightarrow$ LUMO 0.66 & $\mathrm{au} \rightarrow \mathrm{b} 1 \mathrm{~g}$ & YES & \multirow[t]{2}{*}{1.37} \\
\hline & $\mathrm{T}_{2} \mathrm{HOMO}-1 \rightarrow$ LUMO 0.50 & $\mathrm{~b} 2 \mathrm{~g} \rightarrow \mathrm{b} 1 \mathrm{~g}$ & NO & \\
\hline & $\mathrm{T}_{2} \mathrm{HOMO}-2 \rightarrow$ LUMO 0.50 & $\mathrm{au} \rightarrow \mathrm{b} 3 \mathrm{u}$ & NO & \multirow{2}{*}{1.44} \\
\hline & $\mathrm{T}_{3} \mathrm{HOMO}-1 \rightarrow$ LUMO 0.61 & $\mathrm{~b} 1 \mathrm{~g} \rightarrow \mathrm{b} 3 \mathrm{u}$ & YES & \\
\hline \multirow[t]{3}{*}{ Tetracene } & $\mathrm{T}_{1} \mathrm{HOMO} \rightarrow$ LUMO 0.71 & $\mathrm{au} \rightarrow \mathrm{b} 1 \mathrm{~g}$ & YES & \multirow[t]{3}{*}{1.33} \\
\hline & $\mathrm{T}_{2} \mathrm{HOMO}-2 \rightarrow$ LUMO 0.50 & $\mathrm{~b} 2 \mathrm{~g} \rightarrow \mathrm{b} 1 \mathrm{~g}$ & NO & \\
\hline & $\mathrm{T}_{3} \mathrm{HOMO}-1 \rightarrow$ LUMO 0.67 & $\mathrm{~b} 3 \mathrm{u} \rightarrow \mathrm{b} 1 \mathrm{~g}$ & YES & \\
\hline Pentacene & $\mathrm{T}_{1} \mathrm{HOMO} \rightarrow$ LUMO 0.77 & $\mathrm{~b} 2 \mathrm{~g} \rightarrow \mathrm{b} 3 \mathrm{u}$ & YES & 1.37 \\
\hline
\end{tabular}




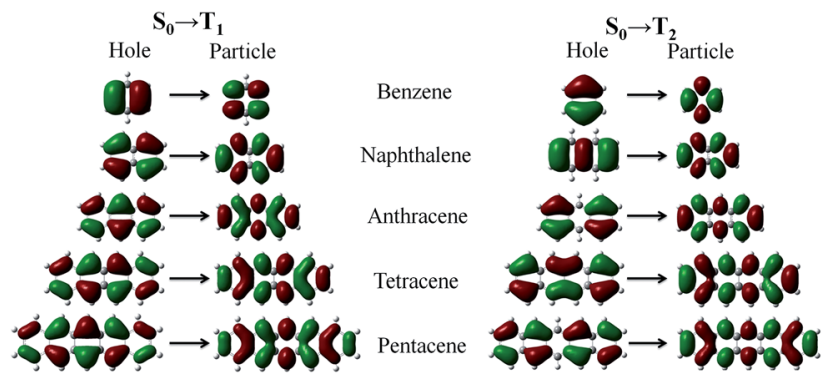

Fig. 5 Natural transition orbital (NTOs) of T1 and T2 for the acenes (benzene, naphthalene, anthracene, tetracene, and pentacene).

(LUMO), and the coefficient increases with the conjugate length. It is implied that the longer the conjugate length, the more intense the transition from the HOMO to LUMO. On the contrary, the transition of the $\mathrm{T}_{2}$ state presents other modes, since it is a transition from the inner MOs of the HOMO to the LUMO. For example the transition of the $\mathrm{T}_{2}$ state in anthracene is HOMO $-2 \rightarrow$ LUMO, tetracene is HOMO $-2 \rightarrow$ LUMO and pentacene is HOMO-1 $\rightarrow$ LUMO and molecules with such transitions are accompanied by a large $\Delta E_{\mathrm{T} 1-\mathrm{T} 2}$ in energy levels. Upon further analysis of the orbital energy, it was noted that the energy differences between HOMO-1 and HOMO in anthracene, tetracene and pentacene are very large, which are $1.28 \mathrm{eV}$, $1.62 \mathrm{eV}$ and $1.44 \mathrm{eV}$, respectively. Therefore, the electron transition energies from these inner occupied orbitals to the LUMO are much larger than from the HOMO to LUMO. We also analyzed the symmetry of the molecular orbital, as listed in Table 1. As is known, the electronic transition is in accordance with the rules of parity, which requires that the molecules must change their symmetry after the transition. In other words, the transition is allowed if the symmetry of the orbital is changed after the electron transition, for example $\mathrm{u} \rightarrow \mathrm{g}$ and $\mathrm{g} \rightarrow \mathrm{u}$, whereas the transition is prohibited for $\mathrm{u} \rightarrow \mathrm{u}$ and $\mathrm{g} \rightarrow \mathrm{g}$. As shown in Table 1, from naphthalene to pentacene, the $\mathrm{T}_{2}$ all show the transition between the same parity MO, which means that these transitions are parity-forbidden. This feature may lead to the emergence of a large energy difference between $\mathrm{T}_{1}$ and $\mathrm{T}_{2}$.

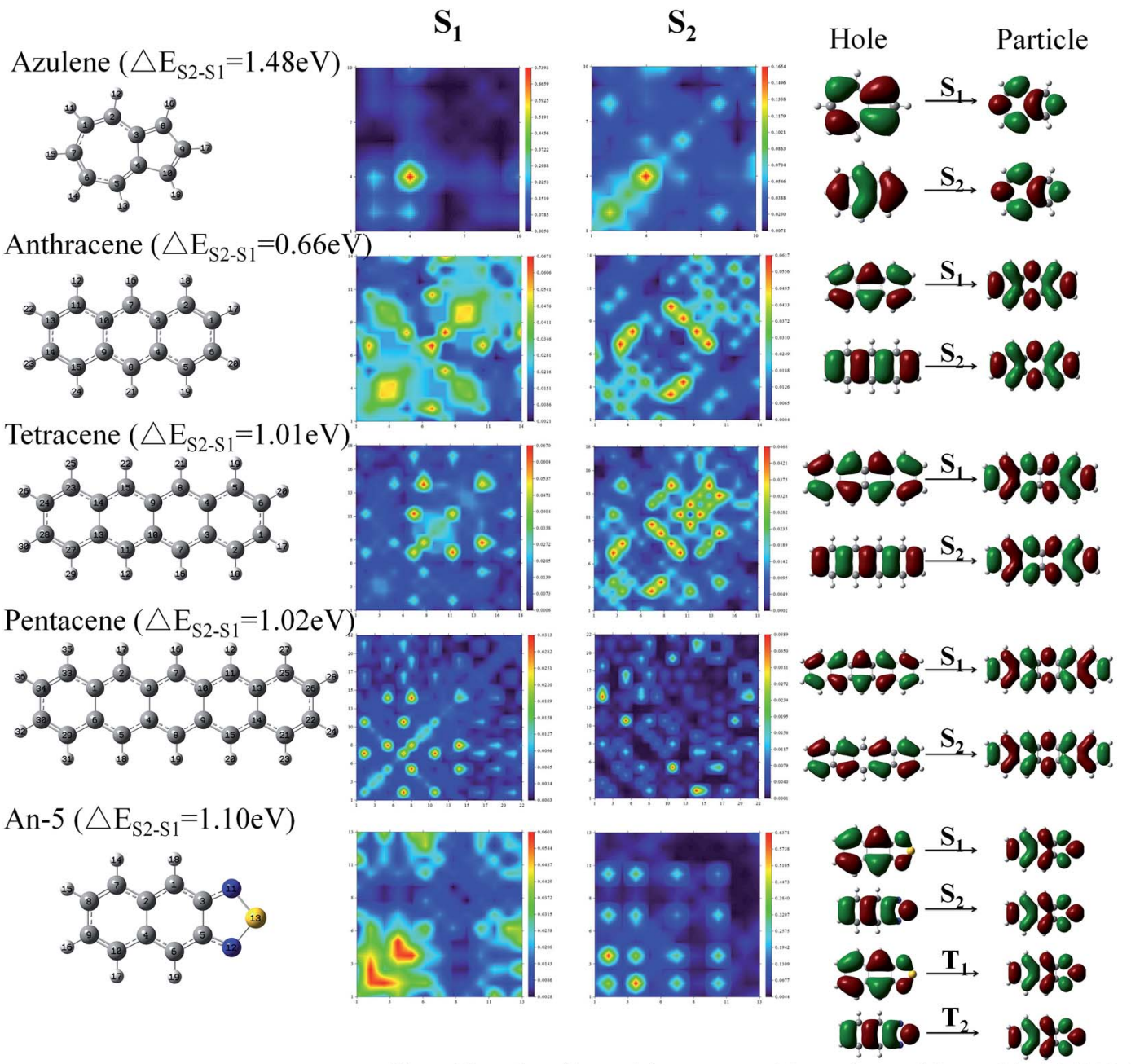

Transition density matrix map Natural transition orbital (NTOs)

Fig. 6 Transition density matrix map and the natural transition orbital (NTO) of $\mathrm{S}_{1}$ and $\mathrm{S}_{2}$ for some molecules (azulene, anthracene, tetracene, pentacene and $A n-5)$, that have a large $\Delta E_{\mathrm{S1}-\mathrm{S2}}$. 


\subsection{Excited state properties}

Excited-state configurations from the stationary points of the ground-state energy surfaces were then obtained at the TD-DFT/ $\omega \mathrm{B} 97 \mathrm{X} / 6-31+\mathrm{G}(\mathrm{d}, \mathrm{p})$ level. We analyzed the natural transition orbital (NTOs) ${ }^{54}$ of the $\mathrm{T}_{1}$ and $\mathrm{T}_{2}$ excited-states of acenes. From Fig. 5 , the nature of the two excited states, $\mathrm{T}_{1}$ and $\mathrm{T}_{2}$, deserves some other comments. In the transition process, the "particles" are very similar but the "holes" are different for $\mathrm{T}_{1}$ and $\mathrm{T}_{2}$. From benzene to pentacene the $\mathrm{T}_{1}$ state is largely represented by singly excited configurations, and among them, the HOMO $\rightarrow$ LUMO has the largest weight. The $\pi \rightarrow \pi^{*}$ density is mostly centered between the $\mathrm{C}$ atoms so that the $\mathrm{T}_{1}$ transition may be classified as a covalent state. On the contrary, the contributions to the $\mathrm{T}_{2}$ state from all singly excited configurations are smaller and more balanced, which correspond to the promotion of an electron from HOMO-1 or HOMO-2 to LUMO, where the location of the electron density distribution changes in different repeat units, such as the charge-transfer state. On the other hand, the NTOs of the $T_{1}$ and $T_{2}$ transition have extremely similar electron cloud distributions with the frontier molecular orbitals (FMOs). The NTOs of the $\mathrm{T}_{1}$ state indicate the character of the local excited state, and the $\mathrm{T}_{2}$ state shows some characteristics of the charge-transfer state. The two excited states show different characteristics of the excited state and the orbital symmetry, thus there is a big difference in energy.

Since the transition of the triplet state is forbidden, we could not calculate the transition matrix, which cannot be intuitive to determine whether the transition properties will affect its energy difference. Hence, for further verification, we selected a few molecules that have large singlet energy differences, $\Delta E_{\mathrm{S} 1-\mathrm{S} 2}$, and quantified the composition of these excited states.

We calculated the wave functions of the electron-hole pairs from the transition density matrix and plotted them in a twodimensional (2D) color-filled map, which is related to the probability of finding the electron and hole in the atomic orbitals localized on each non-hydrogen atom, using the Multiwfn software 3.3.9 Revision. ${ }^{55}$ From this map we can understand which atoms are mainly affected by the electron transition and which atom pairs are strongly coherent when the electron transits. If the effect is stronger during the transition the value is brighter in the map. The diagonal part of the map represents the LE component localized on the main backbone, whereas the off-diagonal region denotes the CT component. The map in Fig. 6 shows that the values of the $S_{1}$ and the $S_{2}$ transition have a great difference, for example, the values of the $\mathrm{S}_{1}$ state of azulene $\left(\Delta E_{\mathrm{S} 1-\mathrm{S} 2}=1.48 \mathrm{eV}\right)$ are only concentrated in the lower left quarter of the map in the diagonal, but the values of the $\mathrm{S}_{2}$ state transition are distributed throughout the graph. Both the diagonal and the off-diagonal are included, which indicates that the transition of the $S_{1}$ state are LE and localized on one of the group of the azulene molecule. On the contrary, the $S_{2}$ state exhibit both CT and LE properties. The same trend is displayed for the other molecules in Fig. 6. This means that the transition properties of the $S_{1}$ state and the $S_{2}$ state are different in mechanism. It is also seen from the distribution of electron clouds of NTOs that the $S_{1}$ and $S_{2}$ states of pentacene and An-5 are the same as that of the $T_{1}$ and $T_{2}$ states, which indicates that the transitions of the $T_{1}$ and $T_{2}$ states also have different transition properties. This may also be one of the reasons that lead to the energy difference.

\section{Conclusions}

The description of the triplet excited state is highly challenging for standard density functionals. Using the typical acenes molecules anthracene, tetracene and pentacene, we benchmarked the EOM-CCSD method and 2 local functionals (SVWN and PBE), 7 hybrid functionals (BLYP, B3LYP, PBE0, BMK, BHHLYP, M06-2X and M06HF), and two long-range-corrected functional CAM-B3LYP and $\omega$ B97X of the DFT method in combination with the $6-31+\mathrm{G}(\mathrm{d}, \mathrm{p})$ basis sets in the framework of the simulation of the triplet excited state properties. The overall results suggest that the $\omega \mathrm{B} 97 \mathrm{X}$ functional is the closest to the experimental values. On the basis of calculating the transition energy of the excited state accurately, we find that the $\Delta E_{\mathrm{T} 1-\mathrm{T} 2}$ of the acenes molecules has a certain trend and anthracene has the largest. We further studied the triplet transition molecular orbital and excited state properties of the acene molecules. The results show that the symmetry of the transition molecular orbital and the excited state properties are very different in the molecule with a relatively large energy gap. This finding will provide an approach for the design of adjustable energy molecules and provide a reference for the application of energy levels of triplet excited states in the future.

\section{Acknowledgements}

We are grateful for financial support from National Science Foundation of China (grant number 51603127, 91233113, 51273078, 51473063, 51203091) and National Basic Research Program of China (973 Program grant number 2013CB834705, 2013CB834801, 2015CB655003).

\section{References}

1 M. Kasha, Discuss. Faraday Soc., 1950, 9, 14.

2 M. Damm, F. Deckert, H. Hippler and J. Troe, J. Phys. Chem., 1991, 95, 2005.

3 P. Foggi, F. V. R. Neuwahl, L. Moroni and P. R. Salvi, J. Phys. Chem. A, 2003, 107, 1689.

4 W. J. Li, D. D. Liu, F. Z. Shen, D. G. Ma, Z. M. Wang, Y. Xu, B. Yang, Y. G. Ma and T. Feng, Adv. Funct. Mater., 2012, 22, 2797.

5 W. J. Li, Y. Y. Pan, R. Xiao, Q. M. Peng, S. T. Zhang, D. G. Ma, F. Li, F. Z. Shen, Y. H. Wang, B. Yang and Y. G. Ma, Adv. Funct. Mater., 2014, 24, 1609.

6 S. Tang, W. J. Li, F. Z. Shen, D. D. Liu, B. Yang and Y. G. Ma, J. Mater. Chem., 2012, 22, 4401.

7 S. T. Zhang, W. J. Li, L. Yao, Y. Y. Pan, B. Yang, Y. G. Ma, F. Shen and R. Xiao, Chem. Commun., 2013, 49, 11302.

8 L. Yao, S. T. Zhang, R. Wang, W. J. Li, F. Z. Shen, B. Yang and Y. G. Ma, Angew. Chem., 2014, 126, 2151. 
9 Y. Y. Pan, W. J. Li, S. T. Zhang, L. Yao, C. Gu, H. Xu, B. Yang and Y. G. Ma, Adv. Opt. Mater., 2014, 2, 510.

10 W. J. Li, Y. Y. Pan, L. Yao, H. C. Liu, S. T. Zhang, C. Wang, F. Z. Shen, B. Yang, Y. G. Ma and P. Lu, Adv. Opt. Mater., 2014, 2, 892-901.

11 Y. C. Luo and H. Aziz, Adv. Funct. Mater., 2010, 20, 1285.

12 C. E. Swenberg and N. E. Geacintov, Org. Mol. Photophys., 1973, 18, 489.

13 M. Pope and C. E. Swenberg, Electronic Processes in Organic Crystals and Polymers, Oxford University Press, Oxford, U.K., 2nd edn, 1999, p. 134.

14 M. Smith and J. Michl, Chem. Rev., 2010, 110, 6891.

15 T. Zeng, R. Hoffmann and N. Ananth, J. Am. Chem. Soc., 2014, 136, 5755.

16 S. F. Nelson, Y. Y. Lin, D. J. Gundlach and T. N. Jackson, Appl. Phys. Lett., 1998, 72, 1854.

17 A. Skotheim and J. E. Reynolds, Conjugated Polymers: Theory, Synthesis, Properties, and Characterization, CRC Press, 2006.

18 K. Müllen and G. Wegner, Electronic Materials: The Oligomer Approach, Wiley-VCH, 1998.

19 J. H. Burroughes, D. D. C. Bradeley, A. R. Brown, R. N. Marks, K. Machay, R. H. Friend, P. L. Burns and A. B. Holmes, Nature, 1990, 347, 539.

20 M. Berggren, O. Inganas, J. Rasmusson, G. Gustafsson, M. R. Andersson, O. Wennerstrom and T. Hjertberg, Nature, 1994, 372, 444.

21 T. Dobbertin, M. Kroeger, D. Heithecker, D. Schneider, D. Metzdorf, H. Neuner, E. Becker, H.-H. Johannes and W. Kowalsky, Appl. Phys. Lett., 2003, 82, 284.

22 G. N. Lewis and M. Kasha, J. Am. Chem. Soc., 1994, 66, 2100.

23 D. S. McClure, J. Chem. Phys., 1949, 17, 905.

24 H. Hunziker, Chem. Phys. Lett., 1969, 3, 504.

25 H. E. Hunziker, J. Chem. Phys., 1972, 56, 400.

26 Y. H. Meyer, R. Astier and J. M. Leclercq, J. Chem. Phys., 1972, 56, 801.

27 S. P. McGlynn, T. Azumi and M. Kasha, J. Chem. Phys., 1964, 40, 507.

28 T. G. Pavlopoulos, J. Chem. Phys., 1972, 56, 227.

29 J. Burgos, M. Pope, C. E. Swenberg and R. R. Alfano, Phys. Status Solidi B, 1977, 83, 249.

30 C. Hellner, L. Lindqvist and P. C. Roberge, J. Chem. Soc., Faraday Trans. 2, 1972, 68, 1928.

31 H. Angliker, E. Rommel and J. Wirz, Chem. Phys. Lett., 1982, 87, 208.

32 G. E. Scuseria and H. F. Schaefer III, J. Chem. Phys., 1989, 90, 3700.

33 Y. Y. Pan, J. Huang, S. T. Zhang, D. W. Yu, B. Yang and Y. G. Ma, RSC Adv., 2016, 6, 108404.

34 M. E. Casida, C. Jamorski, K. C. Casida and D. R. Salahub, J. Chem. Phys., 1998, 108, 4439.
35 R. E. Stratmann, G. E. Scuseria and M. J. Frisch, J. Chem. Phys., 1998, 109, 8218.

36 C. Van Caillie and R. D. Amos, Chem. Phys. Lett., 1999, 308, 249.

37 C. Van Caillie and R. D. Amos, Chem. Phys. Lett., 2000, 317, 159.

38 F. Furche and R. Ahlrichs, J. Chem. Phys., 2002, 117, 7433.

39 S. H. Vosko, L. Wilk and M. Nusair, Can. J. Phys., 1980, 58, 1200.

40 J. P. Perdew, K. Burke and M. Ernzerhof, Phys. Rev. Lett., 1997, 78, 1396.

41 B. Miehlich, A. Savin, H. Stoll and H. Preuss, Chem. Phys. Lett., 1989, 157, 200-206.

42 A. D. Becke, J. Chem. Phys., 1993, 98, 5648.

43 C. Adamo and V. Barone, J. Chem. Phys., 1999, 110, 6158.

44 A. D. Boese and J. M. L. Martin, J. Chem. Phys., 2004, 121, 3405.

45 A. D. Becke, J. Chem. Phys., 1993, 98, 1372.

46 Y. Zhao and D. G. Truhlar, Theor. Chem. Acc., 2008, 120, 215. 47 Y. Zhao and D. G. Truhlar, J. Phys. Chem. A, 2006, 110, 13126.

48 T. Yanai, D. Tew and N. Handy, Chem. Phys. Lett., 2004, 393, 51.

49 J.-D. Chai and M. Head-Gordon, J. Chem. Phys., 2008, 128, 084106.

50 V. A. Rassolov, J. A. Pople, M. A. Ratner and T. L. Windus, J. Chem. Phys., 1998, 109, 1223.

51 H. Chakraborty and A. Shuklab, J. Chem. Phys., 2014, 141, 164301.

52 Y. Y. Pan, J. Huang, Z. M. Wang, D. W. Yu, B. Yang and Y. G. Ma, $R S C A d v ., 2017,7,19576$.

53 M. J. Frisch, G. W. Trucks, H. B. Schlegel, G. E. Scuseria, M. A. Robb, J. R. Cheeseman, G. Scalmani, V. Barone, B. Mennucci, G. A. Petersson, H. Nakatsuji, M. Caricato, X. Li, H. P. Hratchian, A. F. Izmaylov, J. Bloino, G. Zheng, J. L. Sonnenberg, M. Hada, M. Ehara, K. Toyota, R. Fukuda, J. Hasegawa, M. Ishida, T. Nakajima, Y. Honda, O. Kitao, H. Nakai, T. Vreven, J. A. Montgomery Jr, J. E. Peralta, F. Ogliaro, M. Bearpark, J. J. Heyd, E. Brothers, K. N. Kudin, V. N. Staroverov, R. Kobayashi, J. Normand, K. Raghavachari, A. Rendell, J. C. Burant, S. S. Iyengar, J. Tomasi, M. Cossi, N. Rega, J. M. Millam, M. Klene, J. E. Knox, J. B. Cross, V. Bakken, C. Adamo, J. Jaramillo, R. Gomperts, R. E. Stratmann, O. Yazyev, A. J. Austin, R. Cammi, C. Pomelli, J. W. Ochterski, R. L. Martin, K. Morokuma, V. G. Zakrzewski, G. A. Voth, P. Salvador, J. J. Dannenberg, S. Dapprich, A. D. Daniels, Ö. Farkas, J. B. Foresman, J. V. Ortiz, J. Cioslowski, and D. J. Fox, Gaussian 09, Revision D.1, Gaussian, Inc., Wallingford CT, 2009.

54 R. L. Martin, J. Chem. Phys., 2003, 118, 477.

55 T. Lu and F. Chen, J. Comput. Chem., 2012, 33, 580. 